\title{
Technique of goniocurettage: a potential treatment for advanced chronic open angle glaucoma
}

Philipp C Jacobi, Thomas S Dietlein, Günter K Krieglstein

\begin{abstract}
Aim-To introduce a new concept of anterior chamber angle microsurgery, designed to scrape pathologically altered trabecular meshwork from the scleral sulcus as a potential treatment in primary open angle glaucoma.

Methods-Gonioscopically controlled ab interno abrasion of the trabecular meshwork was performed on six human eye banking eyes for morphological analysis. Thereafter, four eyes suffering from terminal glaucomatous optic nerve atrophy as a result of medically uncontrolled intraocular pressure were also treated by 'goniocurettage'. The newly designed instrument resembles a modified cyclodialysis spatula with a bowl-shaped tip, $300 \mu \mathrm{m}$ in diameter, and with its edges sharpened. The treatment zone comprised 4-5 clock hours of the chamber angle circumference.
\end{abstract}

Results-Microscopic examination of the treatment zone revealed that in addition to a complete disruption of the trabecular meshwork and internal wall of Schlemm's canal goniocurettage also caused damage to intracanalicular septa. A splitting along the posterior wall of Schlemm's canal was also noted in one specimen. The clinical data of goniocurettage also showed some promising results. Mean pretreatment IOP averaged 40.7 (SD 8.8) $\mathrm{mm} \mathrm{Hg}$ (range 32-51 $\mathrm{mm} \mathrm{Hg}$ ) and was significantly $(\mathbf{p}<0.04)$ reduced to $18.0(4.2) \mathrm{mm} \mathrm{Hg}$ (12-22 mm Hg) after 6 months, representing an absolute decrease in IOP of 22.7 $\mathrm{mm} \mathrm{Hg}$ and a mean decrease in IOP of $56 \%$. Clinically significant hyphaema occurred in one eye, caused by iatrogenic trauma to a prominent chamber angle vessel. In three eyes a minor reflux of blood occurred at the treatment site. However, no hypotony, choroidal effusion, flattened anterior chamber, or cyclodialysis were observed in these patients.

Conclusion-Morphological analysis of treated postmortem eyes confirmed that goniocurettage completely removed the trabecular meshwork and opened Schlemm's canal, ensuring direct access into the anterior chamber. In a small number of patients over a limited period of time this new surgical procedure resulted in a clinically significant pressure reduction. However, longer term follow up and a greater number of patients are warranted before this experimental procedure is applicable to eyes that would do well with conventional surgery. (Br F Ophthalmol 1997;81:302-307)

Conventional glaucoma filtering surgery is the mainstay of surgical treatment to control intraocular pressure (IOP) in primary open angle glaucoma. ${ }^{1-3}$ There is a growing trend to perform surgery earlier in the course of glaucoma management. ${ }^{4}$ However, despite increasing success rates, especially with the use of adjunct antimetabolites, several problems remain, such as hyphaema, flat anterior chamber, and variable wound healing response to conjunctival manipulation. In order to avoid the latter, various techniques have been investigated that minimise conjunctival dissection to improve the success rate of filtration surgery. Laser sclerotomy has recently become a viable alternative to conventional glaucoma filtration surgery. ${ }^{5}$ However, varying success rates have been reported using different laser systems and techniques. ${ }^{6-10}$ Based on the concept of abnormal resistance to outflow of aqueous humour as a result of maldevelopment of the trabecular meshwork, ${ }^{11}$ goniotomy, ${ }^{12}$ ab externo trabeculotomy, ${ }^{13}$ and trabeculopuncture ${ }^{14}$ have each been recommended as surgical procedures of choice in juvenile open angle glaucoma. In recent years, trabeculotomy has again received increasing interest among some glaucoma surgeons as a first choice surgical treatment of chronic open angle glaucoma, ${ }^{15}$ including combined glaucoma and cataract surgery. ${ }^{16}$

Based on transmission and scanning electron microscopy of trabeculectomy specimens various authors have suggested that in most cases of chronic open angle glaucoma the primary increase of outflow resistance lies in the cribriform layer of the trabecular meshwork adjacent to the inner wall endothelium of Schlemm's canal. ${ }^{17-21}$ Presuming that the outer layers of the trabecular meshwork play the key role in the pathology of primary open angle glaucoma, incisional surgery (goniotomy) or mechanical disruption (trabeculotomy) of the trabecular meshwork could then be a valid surgical approach to medically uncontrolled open angle glaucoma. Unfortunately, simple disruption of the trabecular meshwork with the trabeculotomy approach or punching small holes with the $\mathrm{Q}$ switched Nd:YAG laser 


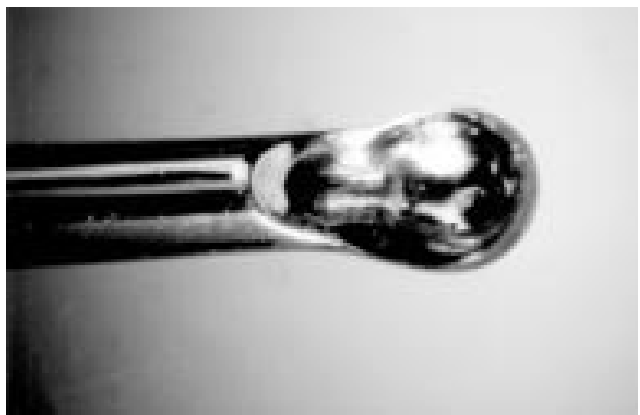

Figure 1 The tip of the 'gonioscraper'. The bowl is 300 $\mu m$ in diameter with its edges sharpened.

(trabeculopuncture) removes little tissue and allows filling in and scarring to occur with subsequent closure of the trabecular opening. ${ }^{22} 23$

The present study was carried out to introduce a new approach in glaucoma surgery aiming to scrape pathologically altered trabecular meshwork off the scleral sulcus in six patients suffering from uncontrolled IOP due to glaucoma absolutum. The aim of the surgical procedure was to abrade rather than incise uveal meshwork; this novel method, therefore, is termed goniocurettage. A description of instrumentation, surgical technique, and preliminary clinical results are given.

\section{Materials and methods}

INSTRUMENTATION AND SURGICAL TECHNIQUE In order to shell the trabecular meshwork out of its scleral sulcus a new surgical instrument was designed. The 'gonioscraper' consists of a small handle and a slightly convex-shaped arm for intraocular use and very much resembles a cyclodialysis spatula. However, the tip of the instrument is shaped as a tiny bowl with 300 $\mu \mathrm{m}$ diameter and with its edges sharpened (Fig 1). In order to abrade clockwise and anticlockwise the scoop is angulated vertically at 90 degrees to the left and right, respectively.

The experimental part of the surgical procedure was carried out on six human eye bank globes, classified unsuitable for keratoplasty. Death had occurred no more than 12 hours

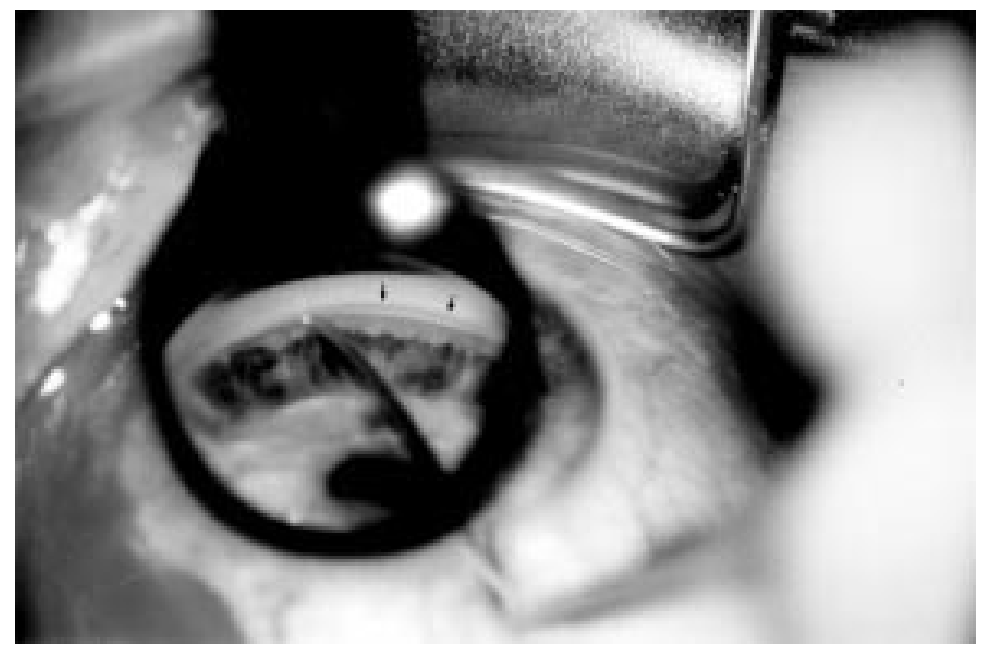

Figure 2 With the aid of an operating microscope and under gonioscopic control ab interno goniocurettage is performed. Following abrasion an irregular pattern of a glistening white band corresponding to the 'denuded' grey-white sulcus scleralis can be seen (black arrows). before surgery. Gonioabrasion was performed under direct visualisation of the anterior chamber angle with an operating microscope and a surgical gonioscopy lens. Following injection of viscoelastic, the 'gonioscraper' was inserted into the anterior chamber through a clear corneal incision at the temporal limbus and directed against the trabecular meshwork at the opposite side. In order to peel off trabecular meshwork the 'scraper' was lightly passed over 2-3 clock hours to either side at the nasal circumference of the anterior chamber angle in sweeping movements (Fig 2). Great care was taken to selectively pare uveal meshwork and not to traumatise adjacent intraocular structures, such as the corneal endothelium or the base of the iris. Gonioscopically, strings of trabecular tissue could be observed intraoperatively to be removed by goniocurettage, leaving a 'denuded' grey-white scleral sulcus. At the end of surgery the viscoelastic along with abraded trabecular debris were removed by means of an irrigation-aspiration probe.

\section{HISTOLOGICAL PREPARATION}

Following surgery three eye banking eyes were processed for scanning electron microscopy as follows: within 5 minutes after treatment, the eyes were immersed in a fixative of $2 \%$ glutaraldehyde and $2 \%$ paraformaldehyde in $0.1 \mathrm{M}$ phosphate buffer at a $\mathrm{pH}$ of 7.4. After 2 hours, the eyes were rinsed in phosphate buffer, and the treated area was dissected out. Specimens for scanning electron microscopy were postfixed with $1 \%$ osmium tetroxide in $0.1 \mathrm{M}$ phosphate buffer at a $\mathrm{pH}$ of 7.4. After 2 hours in osmium tetroxide, the scanning specimens were dehydrated in graded alcohols, critical point dried in carbon dioxide, and sputter coated with gold. The specimens were then examined with the scanning electron microscope. Those samples designated for light microscopy were fixed in a $10 \%$ formalin solution. After 2 hours dissected samples were dehydrated, embedded in paraffin, sectioned by a microkeratome, and stained with haematoxylin and eosin for light microscopy.

\section{PATIENTS}

Six patients were included in this study all suffering from medically uncontrolled IOP, terminal optic nerve atrophy, and no light perception consequent on chronic open angle glaucoma. Exclusion criteria were: reduced $(\geq 20 / 40)$ or threatened vision in the unoperated eye, a history of uveitis, anterior segment media opacity, ocular trauma, and neovascular or angle closure glaucoma. Preoperative evaluation included measurement of visual acuity, quantitative visual field testing if possible, measurement of IOP, gonioscopy, anterior and posterior segment slit-lamp biomicroscopy, indirect ophthalmoscopy of the retina, and ultrasonography when required. Informed consent was obtained from all the patients, following the tenets of the Declaration of Helsinki, after they had been fully informed about the experimental nature of the procedure. Surgery was performed in the above manner using retrobulbar anaesthesia. Treatment in the immediate preoperative period 

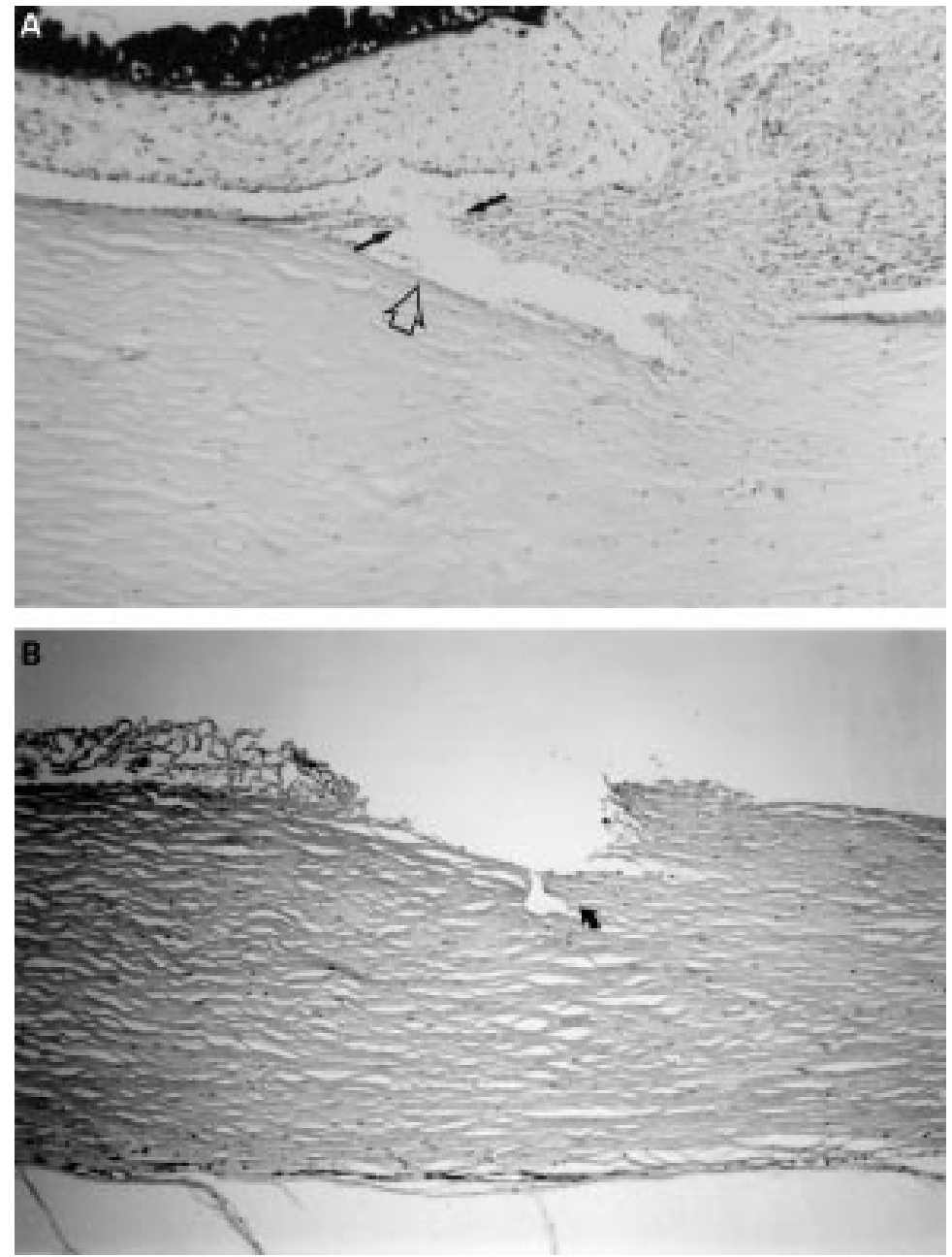

Figure 3 (A) Histological specimen of human anterior chamber angle structures following goniocurettage. The wide iatrogenic defect (black arrow) within the trabecular meshwork and eosin. Magnification $\times 40$. (B) Histological specimen at a greater magnification. Note the complete removal of trabecular tissue in conjunction with damage to the posterior wall of Schlemm's canal. A collector vessel (arrow) is opened. Light micrograph, haematoxylin and eosin. Magnification $\times 100$.

included oral acetazolamide (500 mg), mannitol infusion (Osmofundin, $125 \mathrm{ml}$, intravenously), oculopression for 10-15 minutes, and prophylactic antibiotic drops and ointment.

All IOP readings were obtained with the Goldmann applanation tonometer. Two to four days preoperatively IOP was measured five times a day over a period of 15 hours and the mean was taken as baseline pressure. Postoperatively, regular pressure readings were taken during the period extending to the 12 th month. The same time intervals were used in all patients. The number of postoperative pressure determinations at a specific visit varied from three to as many as five individual determinations for some patients. Analogous mean IOP was taken as a baseline value. Before considering glaucoma surgery and admittance to the present study great care was taken to seek the most effective and tolerable medical treatment for IOP reduction 1-3 months preoperatively (that is, medications being used before surgery were discontinued to find out if they were superfluous). Postoperatively, pressure reducing medication was discontinued in all patients for at least 5 days. Thereafter, recommencement of medical treatment was titrated according to the postoperative pressure measurements. However, there was no switch in type of medication.

\section{Results}

EXPERIMENTAL RESULTS

Goniocurettage performed internally with a newly designed 'gonioscraper' in one third of the chamber angle circumference was gonioscopically controlled under high magnification of an operating microscope. The scraper was observed to pass along near the scleral spur (Fig 2), tending to push trabecular tissue ahead of it, but usually leaving the anterior portion of the trabecular meshwork and Schwalbe's line in place. Gonioscopically, ragged strings of trabecular tissue could be observed intraoperatively to be removed by goniocurettage, leaving an irregular pattern of a glistening white band corresponding to the 'denuded' grey-white scleral sulcus as exposed by histology. From light microscopy of histological sections (Fig $3 \mathrm{~A}$ and $\mathrm{B}$ ) it was evident that in addition to peeling and disruption of the trabecular meshwork the gonioscraper caused damage to septa and endothelium of the external wall of Schlemm's canal, and disruption along the posterior wall of the canal. Flaps of uveal tissue, capable of returning to its predissection position, were not observed in the specimens. Scanning electron microscopy (Fig 4) showed that the trabecular meshwork was pulled from its attachments, leaving ragged structures of Schlemm's canal within the scleral sulcus exposing bare sclera.

\section{CLINICAL RESULTS}

Following its experimental application on human eye bank globes goniocurettage was performed on six patients (two male and four females) with glaucoma absolutum whose ages ranged from 63 to 79 years. Follow up averaged 8.2 (SD 3.5) months (range 4-12 months). Pressure elevation was due to primary open angle glaucoma in five eyes and pseudoexfoliation glaucoma in one eye. The pseudoexfoliative patient was phakic the other five patients were pseudophakic. Goniocurettage was performed over $90-120^{\circ}$ of the chamber angle circumference in all patients. Retreatment was not performed. Preoperative IOP ranged from $32 \mathrm{~mm} \mathrm{Hg}$ to $51 \mathrm{~mm} \mathrm{Hg}$ (mean $40.7 \mathrm{~mm} \mathrm{Hg}$ ). Final postoperative IOP ranged from $12 \mathrm{~mm} \mathrm{Hg}$ to $22 \mathrm{~mm} \mathrm{Hg}$ (mean $18 \mathrm{~mm}$ $\mathrm{Hg}$ ), representing an absolute decrease in IOP of $22.7 \mathrm{~mm} \mathrm{Hg}$ and a mean decrease in IOP of $56 \%$. All patients demonstrated a decrease in IOP (Fig 5), the smallest change being $25 \mathrm{~mm}$ $\mathrm{Hg}$ and the largest being $42 \mathrm{~mm} \mathrm{Hg}$. Postoperatively, the phakic and two of the pseudophakic patients had an IOP less than 19 $\mathrm{mm} \mathrm{Hg}$ with only the pseudoexfoliative patient requiring continued medication with a topical $\beta$ blocker. In one pseudophakic patient, however, the final pressure readings at 10 months postoperatively ranged between 19 and $24 \mathrm{~mm} \mathrm{Hg}$ despite adjunct local antiglaucoma medication. The authors, in accordance with 


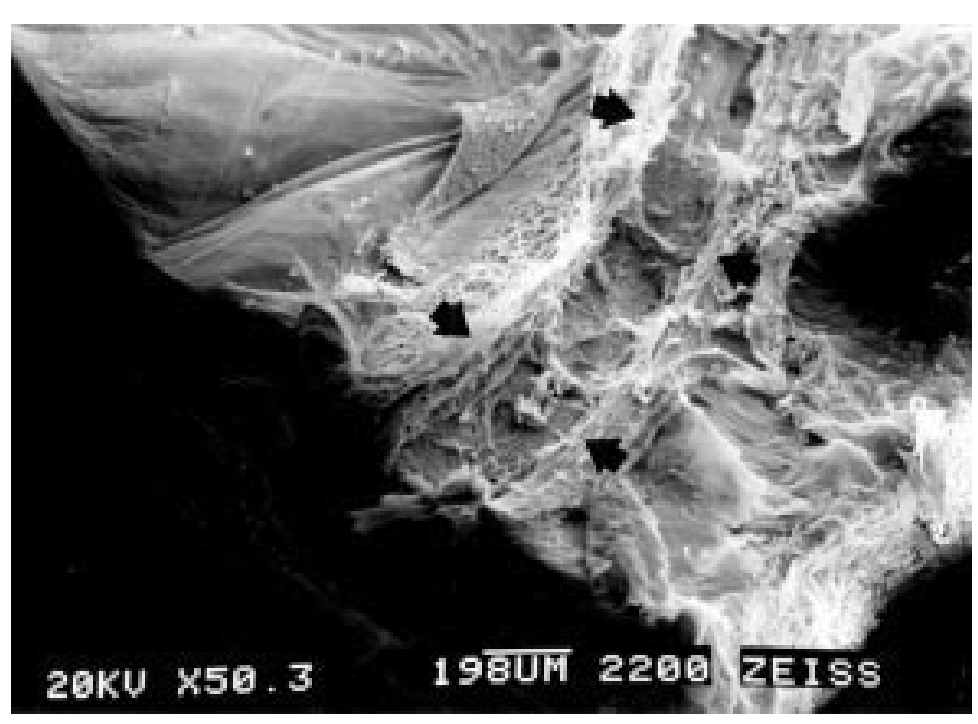

Figure 4 Scanning electron microscopy depicts the deep furrow within the anterior chamber angle, leaving ragged structures of Schlemm's canal within the scleral sulcus. Furrow following treatment in between black arrows.

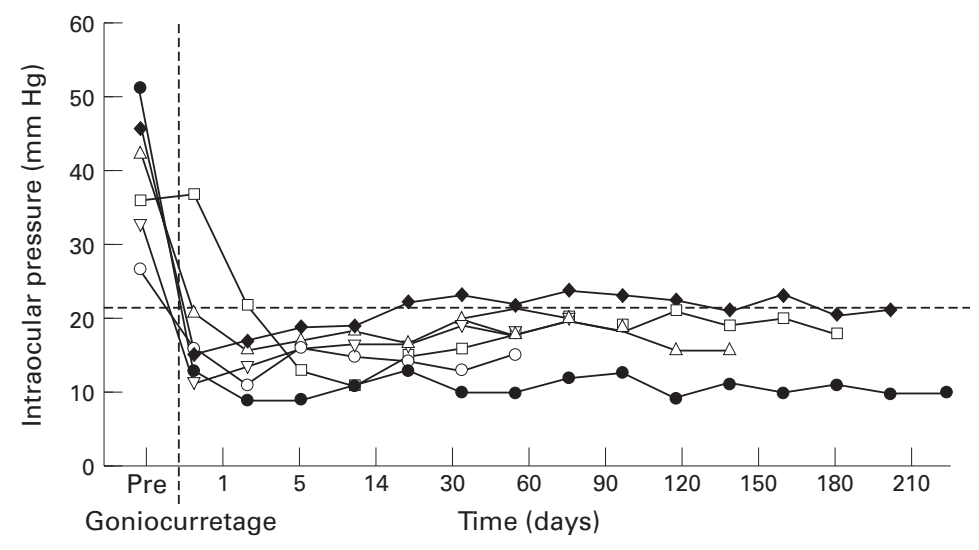

Figure 5 Intraocular pressure of six patients suffering from intractable open angle glaucoma.

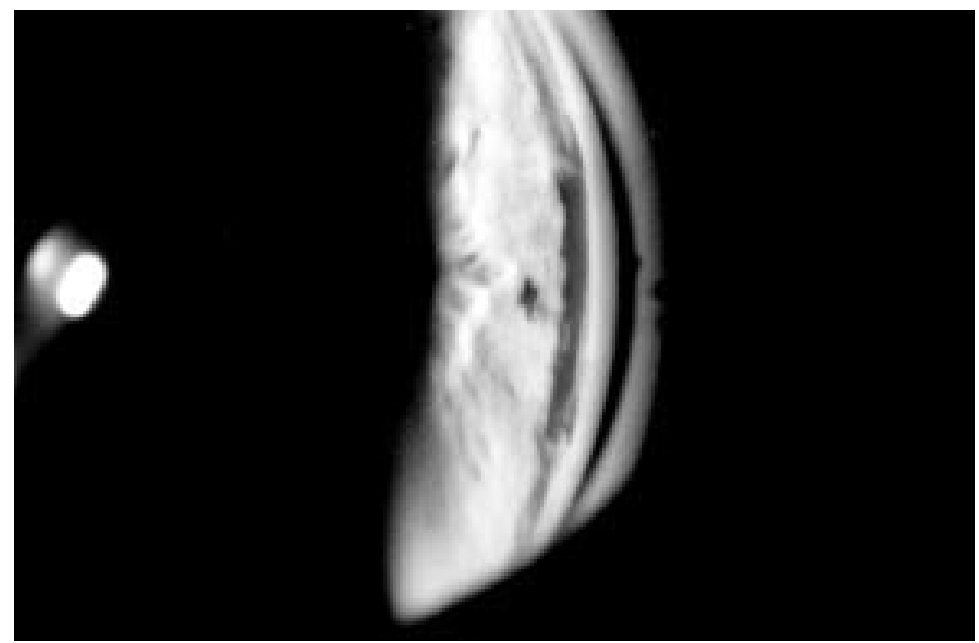

Figure 6 Gonioscopic view of the treatment area following goniocurettage. A small reflux of blood was notified on a regular basis, however, with no further sequelae.

the patient, opted against a retreatment because of a ferre absolutum and reduced general health. All patients were able to discontinue systemic carbonic anhydrase inhibitor postoperatively.
A reflux of blood within the treatment area occurred in four eyes, however, with no further sequelae (Fig 6). In one patient there was significant bleeding into the anterior chamber. This was caused by an iatrogenic trauma to a prominent chamber angle vessel. During the postoperative period the anterior chamber cleared without any further surgical intervention being necessary. In the phakic eye inadvertent descemetolysis of the treatment site occurred. No corneal haze was associated with it. Hypotony (IOP in the $0-5 \mathrm{~mm} \mathrm{Hg}$ range) or choroidal effusion or flattened anterior chamber were not observed. Intraocular inflammation was not prominent after surgery and no patient had a flat anterior chamber.

\section{Discussion}

Microsurgical procedures of Schlemm's canal and the human aqueous outflow system for controlling intraocular pressure in refractory forms of chronic open angle glaucoma have been evolving over the past decades. The basis for most of the present approaches to microsurgery of Schlemm's canal is the finding by Grant, ${ }^{24}$ that the largest portion of resistance to outflow is located within the trabecular meshwork, namely the cribriform layer, ${ }^{18}{ }^{19}$ and can be eliminated by incising the trabecular meshwork and entering Schlemm's canal. ${ }^{25}{ }^{26}$ Attempts to develop surgical approaches are to be found both in conventional and laser surgery. Microsurgical dissection of the trabecular meshwork (trabeculotomy, goniotomy) has again received increasing interest from ophthalmic surgeons, both as treatment for congenital glaucoma as well as for primary chronic open angle glaucoma including combined glaucoma and cataract surgery. ${ }^{15}{ }^{1627-30}$ The procedures themselves have changed little since their original description. ${ }^{31} 32$ Nevertheless, there has been a trend towards newer approaches. With advances in laser technology, many incisional intraocular procedures can now be performed using different kinds of lasers. ${ }^{7101422}$ Gonioscopic, non-contact ab interno laser surgery such as neodymium-YAG laser trabeculopuncture, ${ }^{33}$ short pulsed neodymium-YAG laser trabeculotomy, ${ }^{34}$ neodymium-YLF laser sclerotomy, ${ }^{35}$ and pulsed dye laser sclerostomy ${ }^{36}$ are some good examples of alternative treatment methods using available laser technology. However, incisional surgery as in goniotomy and simple disruption of the trabecular meshwork with the traditional trabeculotomy approach or punching small holes with the Q switched Nd:YAG laser, remove little tissue and allow filling in and scarring to occur with subsequent closure of the trabecular opening. Ito and associates ${ }^{37}$ reported that in monkey eyes 1 year after trabeculotomy, the chamber angle was almost completely restored by newly formed trabecular tissue identical to normal trabecular tissue. At that time no direct communication between Schlemm's canal and the anterior chamber was discernible. In the early postoperative phase, 
however, they observed rather large wound surfaces, possibly facilitating subsequent fibroproliferation and scarring. Thus they came to the conclusion that the observed increase in resistance to aqueous outflow following trabeculotomy is caused by a secondary repair processes of the endothelio-trabecular meshwork closing the sites of earlier trabeculotomy.

In this study we describe a new technique in glaucoma microsurgery aimed at dealing directly with the pathoanatomical site of maximum resistance to aqueous outflow. In an attempt to avoid early reclosure by secondary fibroproliferation of the remaining uveal meshwork goniocurettage removes tissue rather than incising or disrupting the uveal meshwork. In order to do so we designed the 'gonioscraper', a novel microsurgical instrument for transcameral use. Despite the obvious difference in surgical instrumentation the procedure itself, to a certain extent, is comparable with the classic technique of goniotomy. Clear visualisation of the chamber angle structures by gonioscopy and a deep and stable anterior chamber are prerequisites for successful surgical treatment. The intended mechanism of action of goniocurettage is to remove pathologically altered trabecular meshwork and to open a route for aqueous humour to egress either into Schlemm's canal or, in the case of damage of the canal's external wall, to ooze out through microsplittings within the posterior scleral wall.

Microscopic examination of sections of the angles of treated eye bank eyes confirmed that this new technique produced a deep furrow within the trabecular meshwork, completely removing the inner wall of Schlemm's canal. It commonly disrupted intracanalicular septa and damage to the external wall of the Schlemm's canal was also recognisable. Thus, the increase in outflow may not be attributable entirely to the elimination of resistance to flow through the trabecular meshwork and inner wall of Schlemm's canal, but also involve a splitting of sclera posteriorly to Schlemm's canal. This ab interno approach may be an advantageous procedure because the conjunctiva remains undisturbed and, if required, a fistulating procedure can be easily performed at a later point.

A clinical trial evaluating the efficacy of goniocurettage as an antiglaucoma microsurgical procedure in primary open angle glaucoma is currently being carried out. So far, preliminary data from a small group of treated eyes are already available. All six patients experienced an absolute decrease in IOP (mean $22.7 \mathrm{~mm}$ $\mathrm{Hg}$; $56 \%$ from baseline), but IOP in one patient remained significantly greater than 20 $\mathrm{mm} \mathrm{Hg}$. Intraoperatively, the surgical procedure of this phakic patient proved to be more difficult, since extra care had to be taken not to damage the crystalline lens. Surgery was complicated postoperatively by a hyphaema from sustained bleeding of the circulus arteriosus iridis major. Five days postoperatively, the hyphaema almost cleared without further surgical intervention. Undue inflammation was not observed postoperatively in the remaining two eyes. Complications relating to postopera- tive bulbar hypotony or reduced anterior chamber depth did not evolve. Inadvertent cyclodialysis as a mechanism of pressure reduction was ruled out by means of high frequency ultrasound biomicroscopy in all four cases.

However, the limited number of treated eyes and maximum follow up of only 12 months restrict clinical interpretation. At this point goniocurettage has to be regarded as an experimental procedure aiming to relieve trabecular outflow resistance. Longer term follow up and a greater number of patients are warranted before this procedure is applicable to eyes that would do well with conventional surgery.

1 Fluorouracil Filtering Surgery Study Group. Three-year follow-up of the Fluorouracil Filtering Surgery Study. $A m \mathcal{F}$ Ophthalmol 1993;115:82-92.

2 Kawase K, Nishimura K, Yamamoto T, Jikihara S, Kitazawa Y. Anterior chamber reaction after mitomycin and 5-fluorouracil trabeculectomy. A comparative study. Oph5-fluorouracil trabeculectormy.

3 Palmer SS. Mitomycin as adjunct chemotherapy with trabeculectomy. Ophthalmology 1991;98:317-21

4 Jay JL, Murray SB. Early trabeculectomy versus conventional management in primary open angle glaucoma. $\mathrm{Br} \mathcal{F}$ Ophthalmol 1988;72:881-9.

5 Berlin MS, Yoo PH, Ahn RJH. The role of laser sclerostomy in glaucoma surgery. Curr Opin Ophthalmol 1995;6:10214.

6 Latina MA, Dobrogowski MJ. Laser filtration surgery. Ophthalmol Clin N Am 1989;2:625-32.

7 Marshall J. Lasers in ophthalmology: the basic principles.

Eye 1988;2 (Suppl);98-112.
8 Ozler SA, Hill RA, Baervelt G, Andrews JJ, Berns MW. Infrared laser sclerostomies. Invest Ophthalmol Vis Sci 1991;32:58-63.

9 Peyman GA, Badaro RM, Khoobehi B. Corneal ablation in rabbits using an infrared $(2.9 \mu \mathrm{m})$ erbium:YAG laser on rabbits using an infrared $(2.9 \mu \mathrm{m})$ erbium:YAG

10 Hill RA, Baerveldt G, Ozler SA, Pickford M, Prfeta GA, Berns MW. Laser trabecular ablation. Laser Surg Med 1991;11:341-6.

11 Tawara A, Inomata H. Developmental immaturity of the trabecular meshwork in juvenile glaucoma. Am $\mathcal{F}$ Ophthalmol 1984;98:82-97.

12 Ellis $\mathrm{OH}$. The etiology, symptomatology, and treatment of juvenile glaucoma. Am $\mathcal{F}$ Ophthalmol 1948;31:1589-96.

$13 \mathrm{McPherson}$ SD Jr, Berry DP. Goniotomy vs external trabeculotomy for developmental glaucoma. Am f Ophthalmol 1983;95:427-31.

14 Epstein DL, Melamed S, Puliafito CA, Steinert RF. Neodymium-YAG laser trabeculopuncture in open-angle glaucoma. Ophthalmology 1985;92:931-7.

15 Tanihara H, Negi A, Akimoto M, Terauchi H, Okudaira A, Kozaki J, Takeuchi A, et al. Surgical effects of trabeculotomy ab externo on adult eyes with primary open-angle glaucoma and pseudoexfoliation syndrome. Arch Ophthalmol 1993,111:1653-61.

16 Schwenn O, Grehn F, Olbert D. Cataract extraction combined with trabeculotomy. Klin Monatsbl Augenheilkd 1993;203:144-52.

17 Nesterov AP. Role of blockade of the Schlemm's canal in pathogenesis in primary open-angle glaucoma. Am f Ophthalmol 1970;70:691-8.

18 Nesterov AP, Batmanov YrE. Study on morphology and function of drainage area of the eye of man. Acta Ophthalmol 1972;50:337-41.

19 Nesterov AP, Batmanov YrE. Trabecular wall of Schlemm's canal in the early stage of primary open-angle glaucoma. Am $\mathcal{F}$ Ophthalmol 1974;78:639-43.

20 Segawa K. Electron microscopic changes of the trabecular tissue in primary open-angle glaucoma. Ann Ophthalmol 1979;11:49-55.

21 Shabo AL, Reese TS, Gaasterland D. Postmortem formation of giant endothelial vacuoles in Schlemm's canal of monkey. Am f Ophthalmol 1973;76:896-901.

22 Melamed S, Pei J, Puliafito CA, Epstein DL. Q-switched neodymium:YAG laser trabeculopuncture in monkeys. Arch Ophthalmol 1985;103:129-33.

23 Ito S, Nishikawa $M$, Tokura T, Yamane A, Yamagishi K, Miki H, et al. Histopathological study of trabecular meshwork after trabeculotomy in monkeys. Nippon Ganka Gakkai Zasshi 1994;98:811-9.

24 Grant WM. Further studies on facility of flow through the trabecular meshwork. Arch Ophthalmol 1958;60:523-33.

25 Grant WM. Experimental aqueous perfusion in enucleated human eyes. Arch Ophthalmol 1963;69:783-91.

26 Grant WM. Laboratory research. Microsurgery of the outflow channels (symposium). Trans Am Acad Ophthalmol Otolaryngol 1972;76:398-402

27 Deluise VP, Anderson DR. Primary infantile glaucoma (congenital glaucoma). Surv Ophthalmol 1983;28:1-19.
(coluce

28 Anderson DR. Trabeculectomy compared to goniotomy for glaucoma in children. Ophthalmology 1983;90:805-6. 
29 Schwartz AL, Anderson DR. Trabecular surgery. Arch Ophthalmol 1974;92:134-9.

30 Luntz MH, Livingston DG. Trabeculotomy ab externo and trabeculectomy in congenital and adult onset glaucoma. Am f Ophthalmol 1977;83:37-41.

31 Barkan O Technique of goniotomy. Arch Ophthalmol 1938; 19:217-21.

32 Allen L, Burian HM. Trabeculotomy ab externo. Am f Ophthalmol 1962;53:19-26.

33 van der Zypen E, Fankhauser F. The ultrastructural feature of laser trabeculopuncture and cyclodialysis: problems related to successful treatment of chronic simple glaucoma. Ophthalmologica 1979;179:189-200.
34 Lee WR, Dutton GN, Cameron SA. Short-pulsed neodymuim-YAG laser trabeculotomy. Invest Ophthalmol

35 Oram O, Gross RL, Severin TD, Orengo-Nania S, Feldman RM. Gonioscopic ab interno Nd:YLF laser sclerostomy in human cadaver eyes. Ophthalmic Surg 1995;26:136-9. 36 Latina MA, Drobrogowski M, March WF, Birngruber R. Ophthalmol 1990;108:1745-50.

37 Ito S, Nishikawa M, Tokura T, Yamane A, Yamagishi K, Miki H. Histopathological study of trabecular meshwork after trabeculotomy in monkeys. Nippon Ganka Gakkai Zasshi 1994;98:811-9. 